\title{
Audit of the use of psychotropic medication for challenging behaviour in a community learning disability service
}

\begin{abstract}
AIMS AND METHOD
The aims of the study were to identify patients in a community learning disability service receiving psychotropic medication for challenging behaviour, to examine prescribing practice and to compare this against local consensus standards. Local consensus standards were agreed by the consultants and the notes were reviewed by the author.
\end{abstract}

\section{RESULTS}

A total of 102 patients were identified as receiving psychotropic medication for challenging behaviour ( $26.7 \%$ of notes examined). The most common additional diagnoses were autism (29\%) and epilepsy $(28 \%)$. The average duration of treatment was 5.3 years, and multiple drugs were used in $34 \%$ of these patients. Antipsychotics were the most commonly used drugs $(96 \%$ of patients). There was rarely a detailed description of the challenging behaviour. There was little regular monitoring of side-effects or warning about potential side-effects when the medication was started.

\section{CLINICAL IMPLICATIONS}

Challenging behaviour is a common cause of multiple prescribing in learning disability patients, and is often long-term in the absence of a strong evidence base. Other specialties use medication to control disturbed behaviour, particularly in people with dementia or personality disorder, so this audit may also be of interest to old age, adult and forensic psychiatrists.
Emerson (1995) defined challenging behaviour as 'culturally abnormal behaviour(s) of such intensity, frequency or duration that the physical safety of the person or others is likely to be placed in serious jeopardy, or behaviour which is likely to seriously limit use of, or result in the person being denied access to, ordinary community facilities'. In the modified diagnostic criteria for people with a learning disability (DC-LD) published by the Faculty for the Psychiatry of Learning Disability (Royal College of Psychiatrists, 2001), the category of problem behaviours is similar to Emerson's definition, providing operational diagnostic criteria. Research suggests that $12-17 \%$ of people with a learning disability will display challenging behaviour (Kiernan \& Alboraz, 1996), 25\% of people with a learning disability requiring services regularly receive antipsychotic drugs (Branford, 1994) and $48 \%$ of people with a learning disability and challenging behaviour receive antipsychotic medication (Kiernan et al, 1995). The report Valuing People (Department of Health, 2001) expresses concern that 'too often this medication is used as an alternative to adequate staffing'. The use of psychotropic medication to control disturbed behaviour in the absence of mental illness in people with learning disability is a controversial area, not just because of its frequency. Brylewski \& Duggan (1999), in a systematic review, concluded there was no trial-based evidence that antipsychotic medication was effective for challenging behaviour, and that the use of such medication for this purpose could be argued to be unethical outside a randomised controlled trial. There is also evidence that long-term antipsychotic therapy can be successfully withdrawn in a significant proportion of patients (Ahmed et al, 2000).
There are a number of reasons why learning disability psychiatrists prescribe medication for challenging behaviour. These reasons were considered by Bhaumik \& Michael (2004):

'Potential reasons for this include limited resources, lack of clinical psychology input, inability to change environment meaningfully, lack of suitably trained staff to manage private residential homes, pressure from nursing staff and other professionals for immediate resolution of problems. It was acknowledged that many people do not have meaningful employment or day care opportunities.'

Much behaviour may result from maladaptive learning or be communicative, but there does appear to be a biological origin in some cases. There has been much interest lately in the concept of behavioural phenotypes. There is a clear association between some specific causes of learning disability and certain constellations of behaviour that appear to be independent of social and psychological influences, such as severe self-injury in Tay-Sachs disease.

In some cases, extensive psychological input or environmental manipulation may have failed to eradicate a very serious behaviour and it would be hard to refuse to prescribe on theoretical grounds when, for example, someone is self-mutilating on a daily basis. There are no national guidelines about the use of psychotropic medications for challenging behaviour, but Brylewski \& Duggan (1999) suggest that 'target symptoms should be identified, reliably measured and recorded as a baseline before embarking on a therapeutic trial of antipsychotic medication.... If no improvement... results then the antipsychotic should be withdrawn'. Existing research has looked at those receiving services (including social services day care) and did not carefully exclude those receiving medication for mental illness; the medical notes 
were not examined to determine diagnosis or to look at the reasons and justification for prescribing. The audit reported here was limited to those currently receiving

original papers psychiatric input, excluded medication given for mental illness and accessed medical notes to examine the rationale for prescribing.

Antipsychotic medication is frequently used to control behaviour in other specialties, particularly in elderly patients with dementia. This practice has recently come under the spotlight because of concerns about the increased risk of stroke associated with atypical antipsychotics. I would therefore argue that this audit is of interest to all psychiatrists, as the problems are the same whether the patient has a learning disability or not. The objectives of the audit were to:

- identify patients receiving psychotropic medication for challenging behaviour;

- clarify patterns of prescribing;

- compare current practice with agreed standards

- develop interventions to address any problems identified.

\section{Method}

Local consensus standards were agreed within the context of a departmental audit meeting, taking into account recommendations from research. There are no national guidelines concerning the use of medication in challenging behaviour. An audit questionnaire was developed and altered after feedback from the three local consultants and their junior medical staff. All the current notes in Nottingham were sought and examined to identify those receiving psychotropic medication for challenging behaviour, rather than mental illness. In this audit DC-LD categories were used, i.e. verbally aggressive, physically aggressive, destructive, self-injurious, sexually inappropriate, oppositional, demanding and wandering behaviour (Royal College of Psychiatrists, 2001). Patient characteristics were collected from all notes.

\section{Local consensus standards}

The following should be clearly documented in the notes:

1 A clear description of the challenging behaviour, including severity and frequency.

2 Clinical psychology involvement if no response.

3 Annual review of the continued use of medication.

4 Clearly documented benefit from medication.

5 The presence or absence of side-effects at last review.

6 A warning of potential risks before medication started.

Also:

7 No patient should be taking more than one regular drug for challenging behaviour, without clear justification and approval of a post-Membership psychiatrist.

8 Only one regular medication should have been started at a time.

9 Consideration should have been given to withdrawing or reducing the medication in the past 2 years.

10 Patients should preferably not be receiving drugs in dosages exceeding British National Formulary (BNF) limits; patients who are should be appropriately monitored.

\section{Guidelines for completing audit}

The audit source was the handwritten medical notes and clinical letters. Patients included were those receiving psychotropic drugs for behavioural problems; this was determined by the primary reference in the notes being to behaviour, rather than to symptoms of mental illness, and the absence of a diagnosis of mental illness. To meet Standard 1, the nature of the challenging behaviour, its severity and its frequency should have been documented in the past 2 years.

\section{Results}

From 382 sets of notes examined ( $89 \%$ of case-load), 102 patients were found to be receiving regular medication for challenging behaviour (26.7\%). Those receiving when required (PRN) medication only were not included. The author did not find it difficult to distinguish between patients in whom the indication for medication was the control of behaviour and patients receiving medication for the treatment of mental illness. The consultants were provided with a list of their patients identified for the audit, and did not raise any objections to the selection of any of the individuals. Prevalence varied with residence: $46 \%$ of in-patients were receiving medication for challenging behaviour, compared with $36 \%$ of out-patients living in residential care and $16 \%$ living at home. There were 63 men and 39 women and the average age was 39.9 years (range $17-73$ ).

Sixty-four (63\%) of the patients were in the mild to moderate range of learning disability, compared with 38 $(37 \%)$ in the severe to profound range. The most common coexisting diagnoses were autism in 30 patients (29\%) and epilepsy in 29 patients (28\%). A wide range of different classes of drugs were used to control behaviour. Antipsychotic drugs predominated, with 98 patients (96\%) receiving one or more of these agents. Antidepressants, anti-epileptics, lithium, beta-blockers and antilibidinal drugs were also used. The most commonly taken drugs, together with the average daily dose, are listed below:

- 22 patients ( $22 \%$ ) received quetiapine at an average daily dose of $129 \mathrm{mg}$ (range 25-500);

- 21 patients ( $21 \%$ ) received risperidone at an average daily dose of $1.6 \mathrm{mg}$ (range $0.5-4$ );

- 19 patients (19\%) received chlorpromazine at an average daily dose of $186 \mathrm{mg}$ (range 25-600);

- 18 patients (18\%) received zuclopenthixol (including 2 as depot) at an average daily dose of $22 \mathrm{mg}$ (range 450);

- 12 patients ( $12 \%$ ) received haloperidol ( 2 as depot) at an average daily dose of $10 \mathrm{mg}$ (range 1-30).

Patients frequently received anti-epileptic drugs and antidepressants for challenging behaviour. The most common anti-epileptic was carbamazepine in 15 patients $(15 \%)$ at an average daily dose of $733 \mathrm{mg}$ (range 300 


\begin{tabular}{lcc} 
& \multicolumn{2}{c}{ Adherence to standard } \\
\cline { 2 - 3 } Local consensus standard & Percentage & Number of patients \\
\hline The behaviour was described & 98 & $100 / 102$ \\
$\quad$ Nature & 26 & $27 / 102$ \\
Severity & 13 & $13 / 102$ \\
Frequency & 79 & $75 / 95$ \\
2 Clinical psychology input if no improvement & 26 & $24 / 93^{1}$ \\
3 Annual review of continued use of medication & 62 & $63 / 102$ \\
4 Any statement of benefit made & 20 & $19 / 97$ \\
5 Inquiry into side-effects & 8 & $7 / 84$ \\
6 Warning when medication started about risks & 34 & $35 / 102$ \\
7 Polypharmacy & $(22$ patients took two drugs, 8 three drugs, 5 four drugs) \\
& 86 & $30 / 35$ \\
Approval of post-Membership psychiatrist & 29 & $10 / 35$ \\
Clear justification & 99 & $86 / 87$ \\
8 One regular drug started at a time & 49 & $47 / 96$ \\
9 Consideration given to withdrawing or reducing the dosage in the & & $99 / 102$ \\
previous 2 years & 97 & $0 / 3$
\end{tabular}

BNF, British National Formulary.

1. Not all standards applied to all the patients (e.g. medication started recently) and sometimes information was not available in notes (e.g. patient transferred from another health district or hospital decades before).

2. Monitoring should include pulse, blood pressure, temperature, electrocardiogram, urea and electrolytes.

1200). The most common antidepressant was amitriptyline in 9 patients (9\%) at an average daily dose of $54 \mathrm{mg}$ (25-150).

Overall, the average daily dose of antipsychotic for all patients expressed as a percentage of maximum BNF daily dose was 20\% (range 3-160). It was possible to calculate an average duration of treatment for the longestprescribed of the current drugs: this was 5.3 years (range $0-25)$.

The most common types of challenging behaviour described were physical aggression (79\%), self-injury $(42 \%)$, destructiveness (34\%), verbal aggression (26\%), sexually inappropriate behaviour (17\%) and absconsion $(12 \%)$. Benefit was determined by carer impression (89\%), by rating scale $(8 \%)$ and by direct recording of behaviour $(1.5 \%)$. In $1.5 \%$ of cases there was no apparent criterion for the perceived benefit. The adherence to gold standards is described in Table 1.

\section{Interventions}

The following interventions were agreed when this audit was presented at the directorate audit meeting in October 2003:

- a case register of such patients should be compiled to aid future audit;

- record sheets based around the agreed standards should be completed for each patient on an annual basis;

- the audit should be repeated in 6-12 months.

\section{Discussion}

None of the patients met all the local consensus standards. Psychotropic medication is frequently prescribed for challenging behaviour, often for many years, and polypharmacy is common. The frequency and severity of the behaviour are poorly described and the continued use of this medication is not routinely reviewed.

There are no accepted guidelines for using psychotropic medication in challenging behaviour, despite this being common practice. In Nottingham, local consensus standards were agreed, based upon what we considered to be good practice. National (ideally evidence-based) guidelines are needed. There is a need to demonstrate well-considered prescribing characterised by describing behaviour well, considering alternative approaches, using outcome measures, discussing risks with clients and carers and monitoring for side-effects.

\section{Declaration of interest}

None.

\section{References}

AHMED, Z., FRASER, W., KERR, M. P., et BRANFORD, D. (1994) A study of the al (2000) Reducing antipsychotic medication in people with a learning disability. British Journal of Psychiatry, $176,42-46$. prescribing for people with learning disabilities living in the community and National Health Service Care. Journal of Intellectual Disability Research, 38,

BHAUMIK, S. \& MICHAEL, D. M. (2004) Pharmacotherapy and $577-586$

pharmacovigilance in learning disability. Antipsychotic medication for Learning Disability Psychiatry, 6, 9-10. challenging behaviour in people with 
intellectual disability: a systematic review of randomised controlled trials. Journal of Intellectual Disability

original Research, 43, 360-371

papers DEPARTMENT OF HEALTH (2001)

Valuing People: A New Strategy for Learning Disability for the 21st Century. London: Department of Health.

EMERSON, C. (1995) Challenging

Behaviour. Analysis and Intervention in
People with Learning Difficulties.

Cambridge: Cambridge University Press.

KIERNAN, C. \& ALBORZ, A. (1996)

Persistence and change in challenging and problem behaviours of young adults with intellectual disability living in

the family home. Journal of Applied Research in Intellectual Disabilities, $\mathbf{9}$ 181-193.
KIERNAN, C., REEVE, D. \& ALBORZ, A. ROYAL COLLEGE OF PSYCHIATRISTS (1995) The use of anti-psychotic drugs (2001) DC-LD: Diagnostic Criteria for with adults withlearning disabilities and Psychiatric Disorders for Use with challenging behaviour. Journal of Adults with Learning Disabilities/ Intellectual Disability Research, 39, Mental Retardation. Occasional Paper $263-274$. OP48. London: Gaskell.

Tom Marshall Specialist Registrar in Learning Disabilities, Nottinghamshire Healthcare NHS Trust, Byron House, Newark Hospital, Boundary Road, Newark NG24 4DE 